\title{
Arbor
}

\section{Los sistemas de información sanitaria en el marco de un Sistema Nacional de Salud descentralizado}

\section{Vicente Gil Suay}

Arbor CLXXX, 710 (Febrero 2005), 327-342 pp.

\section{Introducción}

Todas las organizaciones de los diferentes sectores productivos del país están realizando un esfuerzo importante para confeccionar e implantar su estrategia, marcando los objetivos que les permitan alcanzar una posición de ventaja en el mercado y conseguir, así, la visión que han definido. Para poner en marcha el despliegue de sus objetivos se ayudan de la identificación de una serie de indicadores que les informarán del grado de consecución de los mismos. Es lo que se conoce como el Cuadro de Mandos de una empresa u organización. Cuando este proceso incorpora objetivos e indicadores estratégicos y no sólo operativos, hablamos, en términos generales, de Cuadro de Mando Integral o Balance Scorecard (CMI o BSC). Los indicadores se convierten así en elementos transcendentales que toman «el pulso» diario a la evolución de la empresa. El sistema de información es el que facilita la obtención de los indicadores, su transmisión, análisis, comparación, etc., utilizando diferentes herramientas informáticas (software) para realizar estas tareas. Además garantiza la calidad, transparencia, vigencia, coherencia y disponibilidad. de sus indicadores.

El Sistema Nacional de Salud (SNS) es la organización encargada de promover, mantener y recuperar el estado de salud de los ciudadanos de nuestro país y de aquellos que aún no siendo ciudadanos españoles, tienen derecho a las prestaciones sanitarias. Como organización, el SNS se 
debe dotar de una estrategia que asegure la consecución de lo que en términos de gestión conocemos como la visión, y establecer, primero, los objetivos estratégicos y, después, los indicadores que informen al sistema sobre la consecución de los objetivos.

Hasta aquí podríamos decir que en nada difieren las dificultades que tienen las organizaciones de otros sectores de las que tiene el SNS para establecer sus indicadores y los sistemas de información que los soportan. Sin embargo, la realidad política de nuestro país introduce un elemento diferenciador importante en el ámbito sanitario ya que nuestro SNS se sustenta sobre una estructura descentralizada compuesta por 17 Comunidades Autónomas (CA) con competencias sanitarias y un Ministerio de Sanidad y Consumo (MSC) que ha visto disminuir sus competencias de manera notable al finalizar el proceso transferencial, quedando éstas limitadas, en gran medida, a labores de coordinación entre las diferentes autonomías.

En este contexto es fácil pensar que cada comunidad autónoma establece su propia estrategia, objetivos e indicadores que no necesariamente serán similares a los de otra comunidad. A esta falta de homogeneidad de los diferentes indicadores del sistema, o ¿de los 17 sistemas?, se une la escasa o nula disponibilidad de dichos indicadores, tanto para los ciudadanos (contribuyentes) como para los profesionales del sector, lo que impide la evaluación, comparación y aplicación de políticas de mejora.

\section{Los antecedentes de la situación}

Nuestro sistema sanitario, antes de la instauración de la democracia y, por supuesto, antes de la descentralización, tenía divididas sus competencias en varios departamentos sanitarios: las de Salud Pública en una Dirección General de Sanidad dependiente del Ministerio de Gobernación (o de Interior); los servicios sanitarios asistenciales agrupados bajo el Instituto Nacional de Previsión (INP, posteriormente INSALUD) dependiente del Ministerio de Trabajo y un tercer grupo constituido por los centros sanitarios dedicados a la lucha contra enfermedades especiales como la lepra, tuberculosis, etc., que se integraban bajo la llamada Administración Institucional de la Sanidad Nacional (AISNA) también dependiente del Ministerio de Gobernación-Interior.

Con la creación del Ministerio de Sanidad y Seguridad social en la era democrática (1977) se unifican los tres grupos de competencias citados. Sin embargo, poco tiempo después, en 1979, comienza a cederse a las CA competencias en materia de Salud Pública y de la gestión del AISNA. An- 
tes de la entrada en vigor de la Ley General de Sanidad y por tanto de la creación del SNS, ya se habían transferido competencias sanitarias a varias $\mathrm{CA}$. Y es que la transferencia de los servicios sanitarios a las CA obedece más a razones de índole política y necesidad de desarrollo autonómico, que a necesidades de planificación sanitaria. Esto ha condicionado, incluso, un diferente ritmo transferencial que se inicia antes de la Ley General de Sanidad (1986) con las llamadas comunidades históricas y que ha culminado recientemente (2002) con la transferencia simultánea de competencias a diez CA que todavía eran gestionadas por el antiguo INSALUD. EI carácter marcadamente político de este proceso hace difícil plantear la reversión de las competencias, aún en el supuesto de una disfuncionalidad de la distribución de competencias efectuada. No es razonable pensar que las CA cedan en un futuro competencias a favor de una gestión centralizada.

Así las cosas, no resulta difícil comprobar que la disponibilidad de los indicadores y de los sistemas de información, tanto en el ámbito central como autonómico, es escaso en este periodo y tampoco responden a la lógica que la existencia de una estrategia impone a dichos indicadores.

\section{Situación actual}

A mediados de la década de los 90 es notoria la necesidad de avanzar y mejorar en la elaboración de los indicadores del SNS y de los sistemas de información que los soportan, pero se hace evidente las dificultades que en un entorno de sanidad descentralizada representa esta tarea. La necesidad de culminar el trabajo transferencial al final de dicha década fija las prioridades del Ministerio, en detrimento del necesario establecimiento de instrumentos de coordinación en paralelo al proceso de descentralización y no resulta un ambiente propicio para el desarrollo de indicadores estratégicos del SNS.

Acabado el proceso de transferencias en 2002 se hace patente de una forma meridiana la necesidad de dotar al SNS de la suficiente cohesión que garantice la equidad del sistema, asegurando el acceso a las prestaciones y el derecho a la salud en condiciones de igualdad en todo el territorio nacional, la calidad en las prestaciones y la necesaria participación ciudadana que posibilite la consideración de sus expectativas y el respeto a la autonomía de sus decisiones. De esta forma nace la Ley de Cohesión y Calidad del SNS el 29 de Mayo de 2003 que contó con el voto favorable de todos los grupos parlamentarios del Congreso sin excepción. 
Con esta Ley se dota al SNS por primera vez de una serie de estructuras que garanticen un sistema de información completo y de calidad que permita el análisis de los diferentes indicadores, la comparación de resultados y, en definitiva, la introducción de la mejora continua en el sistema. En su artículo primero establece el objeto de la misma que no es otro que el de establecer un marco legal para las acciones de coordinación y cooperación de las Administraciones públicas sanitarias de modo que se garantice la equidad, la calidad y la participación social en el SNS, así como la colaboración activa en la reducción de las desigualdades en salud. Dichas acciones comprenden, entre otras: los sistemas de información y la calidad del sistema sanitario.

La Ley establece en su capítulo V las características y requisitos del sistema de información que garantizará la disponibilidad de la información y la comunicación recíproca entre las Administraciones sanitarias. Encarga al Consejo Interterritorial (CI) la fijación de objetivos y contenidos de dicha información, aspecto éste de suma trascendencia. El sistema de información sanitaria (SIS) contendrá información, entre otros aspectos, de: datos poblacionales, recursos humanos y materiales del sistema, actividad desarrollada y resultados obtenidos. EI MSC, previo acuerdo del CI, establecerá la definición y normalización de datos y flujos, la selección de indicadores y los requerimientos técnicos necesarios para la integración de la información. Esta tarea de definición y normalización de los datos es fundamental, pues sin ella resultaría imposible garantizar la suficiente calidad del sistema y haría imposible la evaluación y, por tanto, la aplicación de medidas correctoras. Señala la norma que los usuarios del SIS serán las Administraciones Públicas Sanitarias, los gestores y profesionales de la salud y los ciudadanos, obviamente, bajo el cumplimiento de la legislación en materia de protección de datos de carácter personal. Se prevé para el uso de esta información la utilización de una red segura de comunicaciones que facilite y dé garantías de protección al intercambio de información entre sus integrantes.

En este capítulo también se hace mención a dos componentes importantes de la información sanitaria como son los datos clínicos y de salud individual y la tarjeta sanitaria. En cuanto a los primeros, se hace mención a la necesidad de permitir su acceso tanto a los interesados como a los profesionales que participen en su asistencia sanitaria y atribuye al MSC el establecer un procedimiento que permita el intercambio telemático de la información. Sin embargo, en estos momentos la ausencia de dicho procedimiento contrasta con el hecho de que no son pocas las CA que están aplicando diferentes modelos de historia electrónica, tanto en atención especializada como primaria, sin que se pueda acceder a su conteni- 
do desde un centro sanitario de otra CA. Es decir, que los datos clínicos de un paciente con historia electrónica en una CA resultan opacos para el médico que le atiende en otra CA como desplazado, con la consiguiente pérdida de calidad de la atención prestada. Algo similar sucede con la tarjeta sanitaria que, aunque disponible ya en algunas CA, no lo esta en otras y, en aquellas en que existen, los datos que contienen no son homogéneos ni accesibles desde otra CA diferente a la propia. Del mismo modo que para la historia electrónica, el intento de normalizar este instrumento llega con retraso y supondrá un esfuerzo añadido al ya efectuado y con costes adicionales nada despreciables. Demasiadas ineficiencias para un entorno de racionalización del gasto sanitario.

Estos dos ejemplos sirven perfectamente para entender la débil situación en la que se encuentra nuestro SNS en cuanto a los SIS se refiere, ya que pone sobre la mesa las carencias en materia de coordinación y de estrategias comunes entre las diferentes Administraciones Públicas Sanitarias. Es más, en algunos casos existe un desmesurado afán diferenciador de unas CA respecto de otras, anticipándose a la necesaria coordinación entre ellas con el sólo propósito del rédito político. Llama la atención este comportamiento con los que encontramos en otros países de nuestro entorno con SNS como es el caso de la estrategia definida por el National Health Sistem (NHS) para la incorporación de las tecnologías de la información en los próximos diez años. Para el Departamento de Salud británico, la información y la comunicación en el NHS necesitan ser gestionadas y controladas en el ámbito nacional dado el papel estratégico que juega en la prestación sanitaria. No es un programa de tecnologías sino una forma diferente de trabajar y de prestar la asistencia. ¡Contará con una inversión para el periodo antes mencionado de nueve mil millones de euros!. El carácter integrado del plan y la fuerte financiación del mismo auguran, a priori, un éxito importante.

Volviendo a nuestro país, la ley contempla tres nuevos organismos de carácter central para reforzar el sistema de información, la calidad del SNS y prestar apoyo al CI. Estos son, el Instituto de Información Sanitaria, la Agencia de Calidad del SNS y el Observatorio del SNS.

El Instituto se encarga, fundamentalmente, de recabar, elaborar y distribuir la información que necesita el SNS de acuerdo con las directrices que establezca el CI.

Entre las funciones de mejora encargadas a la Agencia de Calidad del SNS destacan la elaboración de normas de calidad y seguridad, de indicadores que permitan comparar la calidad de los diversos centros y servicios, de guías de práctica clínica y del registro de buenas prácticas y de acontecimientos adversos. 
El Observatorio del SNS, dependiente del MSC, debe proporcionar un análisis permanente del SNS en su conjunto mediante estudios comparativos de los servicios de salud de las $\mathrm{CA}$, de la provisión de servicios, de la gestión sanitaria y de los resultados. También realizará funciones de análisis de las reformas sanitarias internacionales que puedan ser de interés al SNS.

La creación de estas tres estructuras abre una puerta de esperanza hacia la mejora de los SIS del SNS aunque convendrá delimitar mejor las funciones de alguno de ellos ya que se advierten solapamientos sobre el manejo y comparación de los indicadores.

En este entorno descentralizado el CI debe jugar un papel sólido, serio y responsable si queremos que se mantenga el principio de equidad que caracteriza a nuestro SNS. En lo referente a los SIS, el CI establecerá los criterios, sistemas y medios de relación que permitan la información recí proca en el SNS, otorgando así al Consejo un protagonismo absoluto en este apartado. Sin embargo, las actuaciones del mismo se circunscriben al nivel de recomendaciones dado el papel que nuestra Constitución otorga al régimen autonómico, en el que cada CA, mantiene la independencia de sus decisiones en materia sanitaria. Los distintos avatares por los que esta transcurriendo esta importantísima institución no permite ser opti mistas a la hora de esperar de ella una estrategia común por parte de las diferentes CA que la componen y, por ende, no permite ser optimistas a la hora de contar con un SIS que responda a las necesidades del despliegue de dicha estrategia. Como ejemplo basta citar las vicisitudes por las que ha transcurrido la información de las listas de espera quirúrgicas. Convertidas en materia de confrontación política que no de debate constructivo, ha sido sumamente laborioso llegar, en esta materia, a un consenso que permita ofrecer a los ciudadanos una información homogénea y de calidad. Si se ha tenido que recorrer tan largo periplo para un solo indicador, alcanzar el necesario consenso para el resto parece una tarea casi imposible. La excesiva politización de la gestión sanitaria lastra en exceso el necesario progreso de nuestro SNS que todavía goza de buena salud pero al que habrá que cuidar en el futuro si queremos que no pierda la privile giada posición internacional que ahora ocupa. Estos hechos y los importantes retos a los que nos enfrentamos, entre otros la financiación deficitaria del SNS, el incremento de costes por el incremento del consumo y el encarecimiento de las nuevas tecnologías, la deficitaria prestación sociosanitaria, etc., hacen necesario un pacto político por la sanidad como se demanda desde diferentes estamentos de nuestra sociedad.

Las nuevas tecnologías permiten la transferencia de información mejorando de forma notable la homogeneización de ésta, independiente- 
mente de la fuente de origen. Este es el caso de los lenguajes XML o la invocación remota mediante webservice. Pero además, se debe realizar una tarea importante orientada a la utilización de estándares de comunicación que permitan, precisamente, el intercambio de ésta información, como por ejemplo, en el caso de la historia electrónica de salud, el uso de estándares HL7, CEN o DICOM.

A continuación podemos ver algunos ejemplos de la información disponible en Internet, una de las herramientas más potentes para la información y empleada por todas las entidades que componen el SNS.

\section{Website del Ministerio de Sanidad y Consumo}

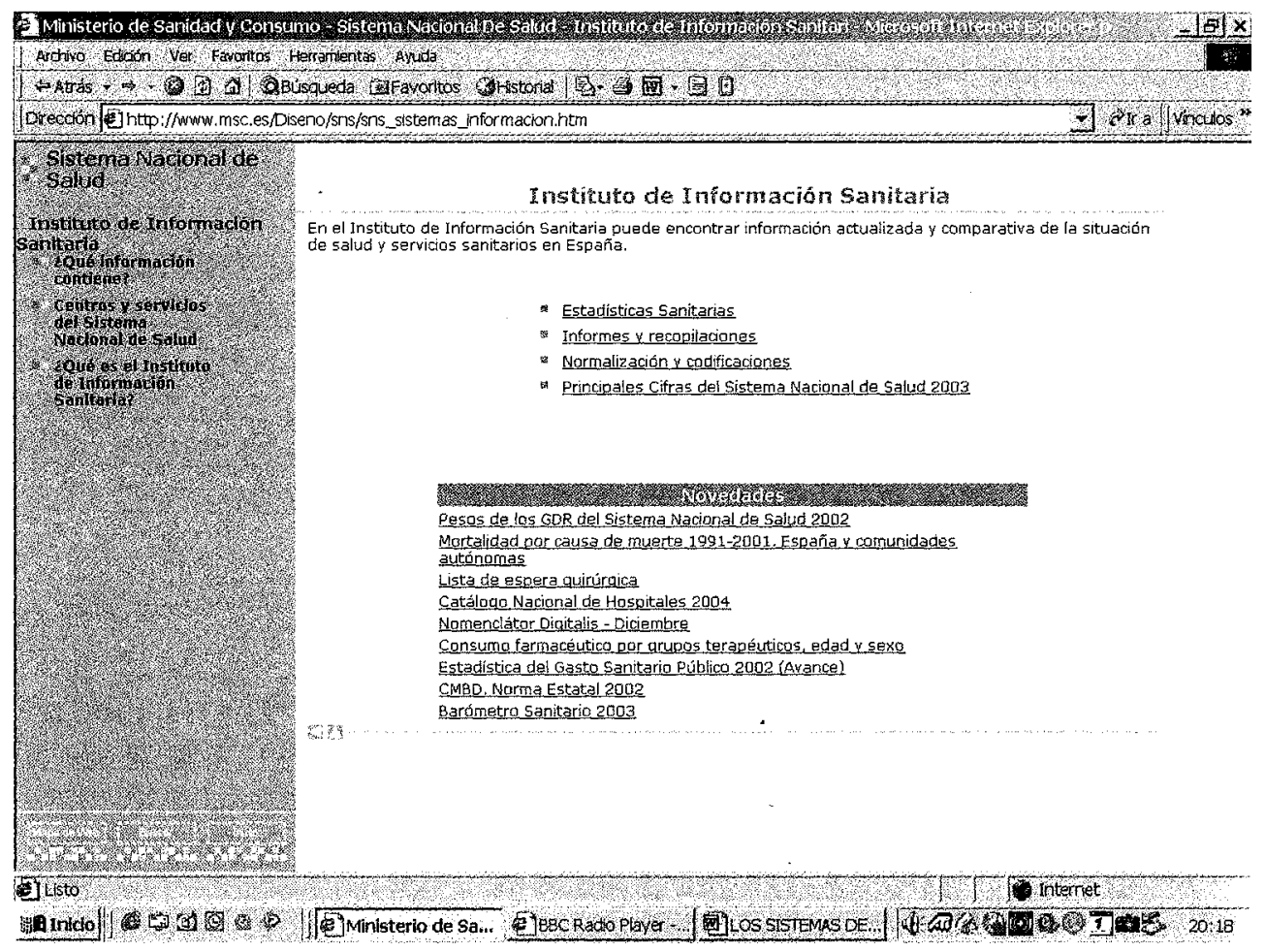

Fig. 1

En esta imagen (Fig.1) podemos ver la página de acceso que el MSC destina al Instituto de Información Sanitaria y los contenidos de la misma. Si nos adentramos en algunos de los indicadores (Fig.2) obtenemos, 
por ejemplo, información sobre el gasto sanitario publico, en donde podemos obtener información acerca del gasto sanitario público y privado y porcentaje respecto del PBI. En la siguiente figura (Fig.3) podemos ver la entrada a la información de Lista de Espera Quirúrgica (LEQ).

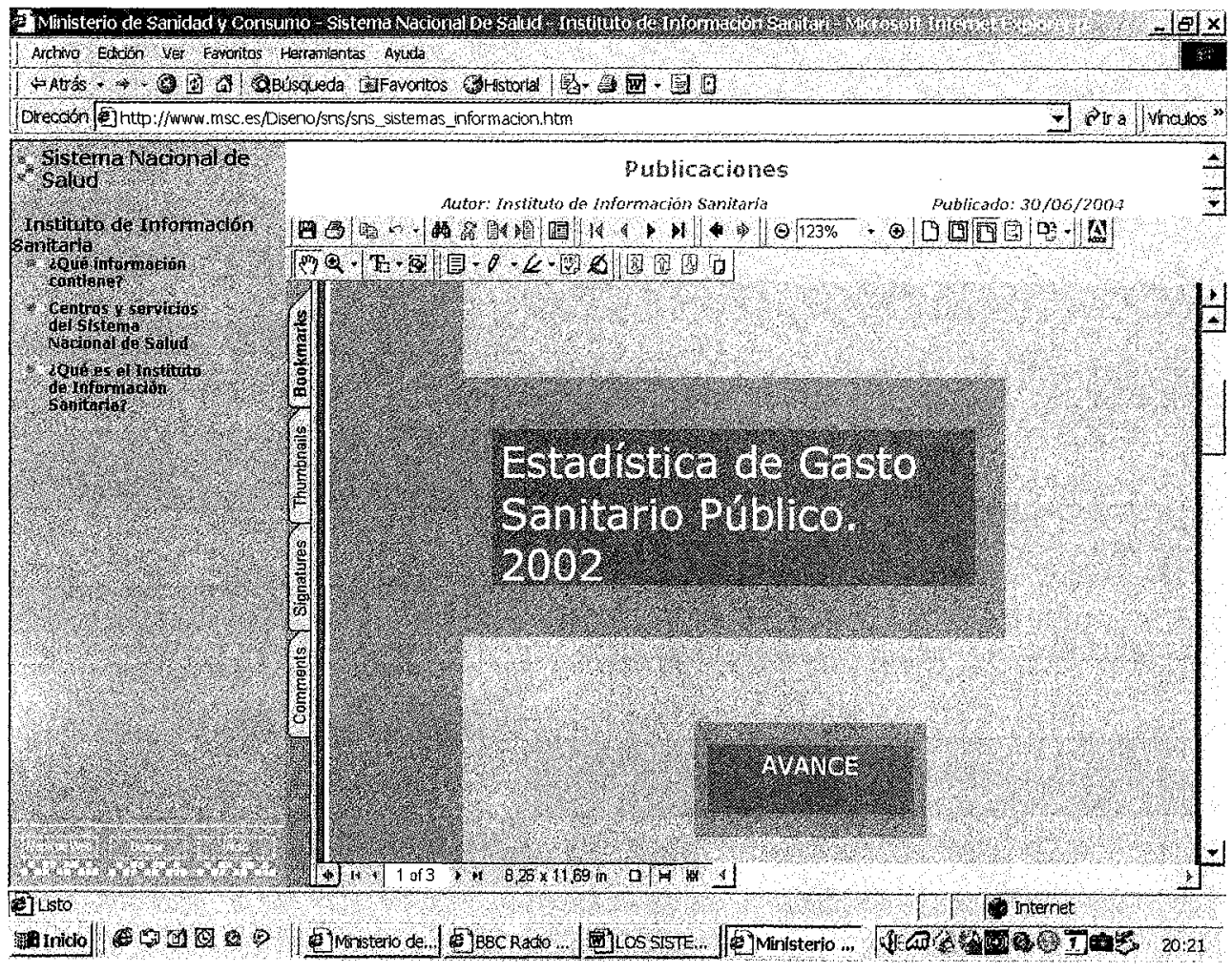

Fig. 2

Merece la pena hacer algún comentario respecto a estos indicadores. En principio, al observar el contenido de la página de acceso uno podría esperar que el apartado "Gasto Sanitario» contuviera un gran número de indicadores referente a esta área. Sin embargo, la realidad es que contiene una información preliminar y, además, referida al año 2002, es decir con dos años de retraso e incompleta. Pero en cualquier caso si vemos la información de 2001 observamos que el gasto del SNS está desagregado en una serie de indicadores como el porcentaje de gasto de personal, gasto corriente, o bien el gasto de la atención especializada o primaria. Siendo estos datos importantes no cabe duda que se enriquecería la información si estos mismos indicadores estuvieran desagregados en el ámbito de CA, o incluso poder llegar a conocer niveles de gasto por áreas sa- 


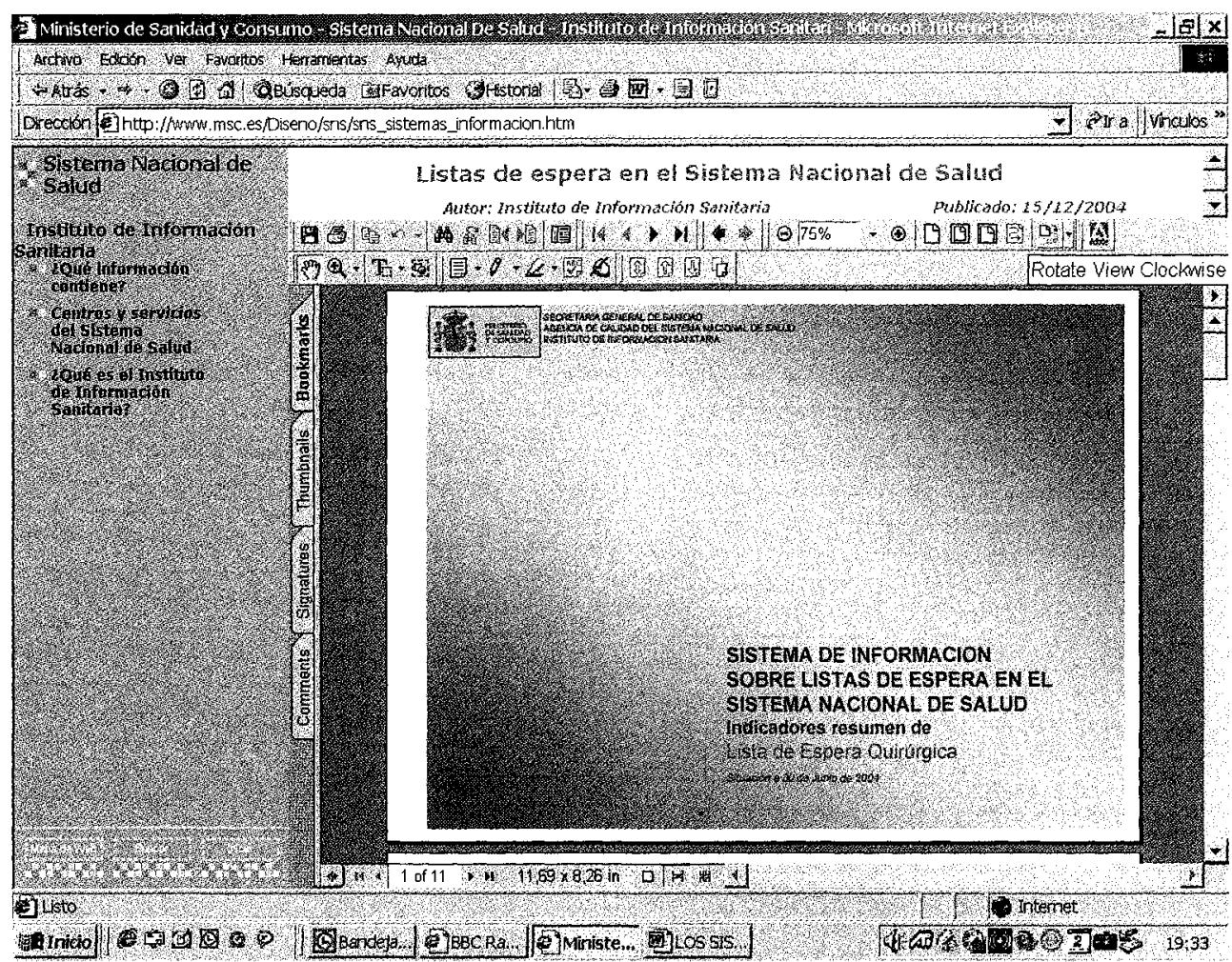

Fig. 3

nitarias o el gasto real medio por habitante, lo que permitiría una comparación entre diferentes áreas y CA. En cualquier caso, lo que se advierte es que los indicadores no provienen de una selección de éstos basada en la estrategia sino que responden a otro tipo de necesidades, o tal vez incluso a la posibilidad o no de obtener una información actualizada y completa, o que cuente con el consenso de las diferentes CA para su publicación.

La información de la LEQ sí se encuentra actualizada a fecha 31 de Junio de 2004 y sus contenidos sí están en relación con una decisión estratégica pues, por ejemplo, contienen datos de espera por especialidades y procesos más problemáticos en la espera, número de pacientes en espera, demora media, etc. En este caso hay que señalar que la información proviene de un acuerdo previo del CI y además se ha elaborado de forma homogénea para todo el territorio nacional. A pesar de estas mejoras en los contenidos, los ciudadanos, los contribuyentes por tanto, no pueden conocer, al menos por ahora, la información por CA ni los tiempos de es- 
pera máximos, etc.. No resulta fácil explicar por qué no esta disponible esta información si entendemos que nuestro SNS esta orientado a los ciudadanos. No estamos respondiendo, en este caso, a las necesidades de información que la sociedad plantea y que la Ley de Cohesión y Calidad reconoce y quiere atender.

En la siguiente figura (Fig. 4) podemos ver datos del catálogo nacional de hospitales. Como en los casos anteriores, podría enriquecerse notablemente la información si encontrásemos, además del contenido actual, datos sobre recursos, actividad y resultados de los diferentes hospitales, estratificados, por ejemplo por niveles de complejidad, número de camas, centro docente y/o universitario, etc.

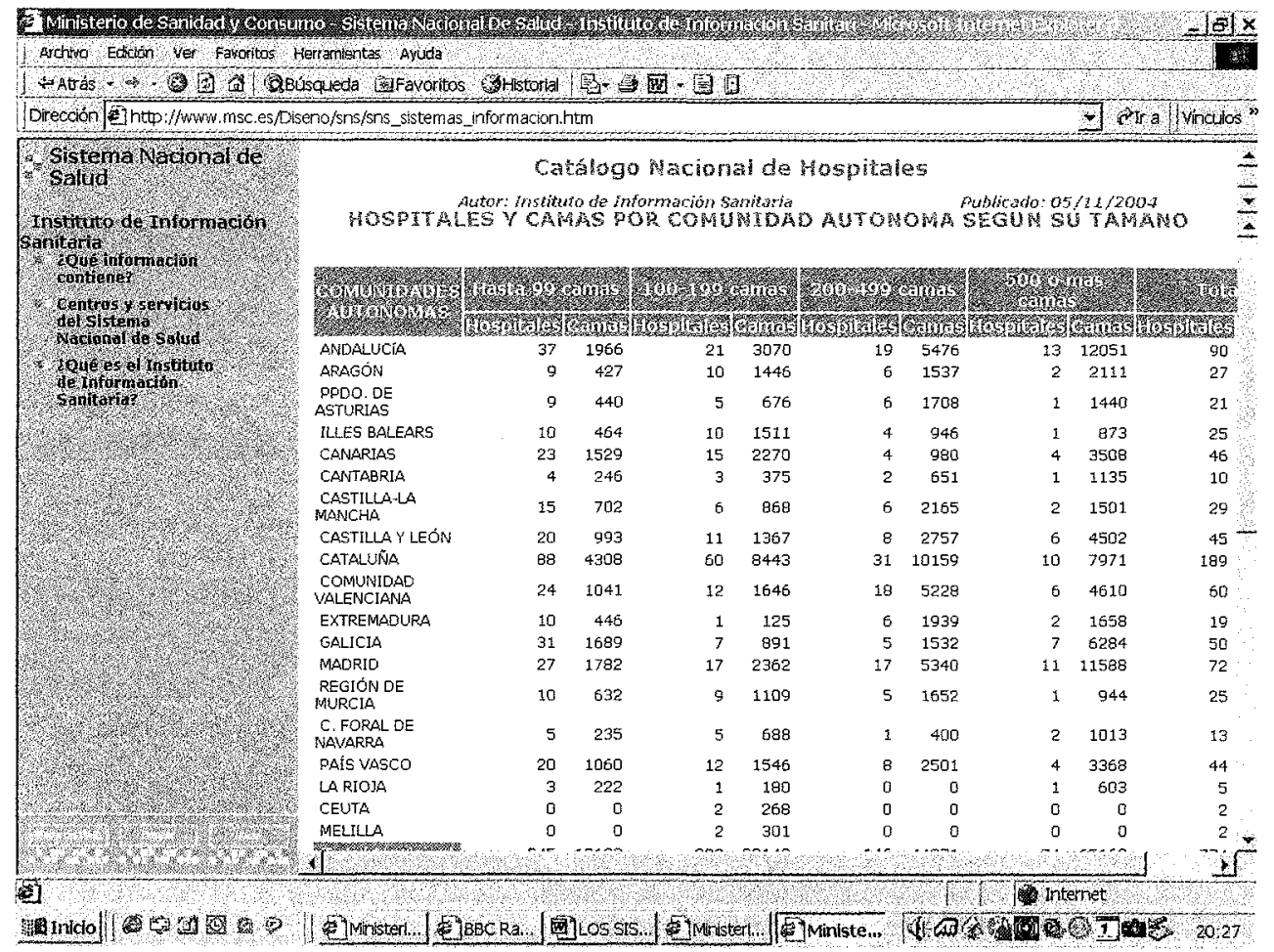

Fig. 4

De lo visto en estos ejemplos, y que se puede revisar si se accede a www.msc.es, podemos concluir que aún contando con un buen número de indicadores cualitativamente importantes, su actualización no es la deseable, la estructura de la información no responde a las necesidades que los cuidadnos plantean, ni tampoco a las de los responsables autonómi- 
cos o de los centros de gestión del SNS y, además, se imposibilita la comparación de los resultados, elemento éste fundamental para la mejora continua. En definitiva, sus contenidos no están al servicio de una estrategia.

\section{Website de Comunidades Autónomas} CA.

Veamos algunos ejemplos de la información disponible en algunas

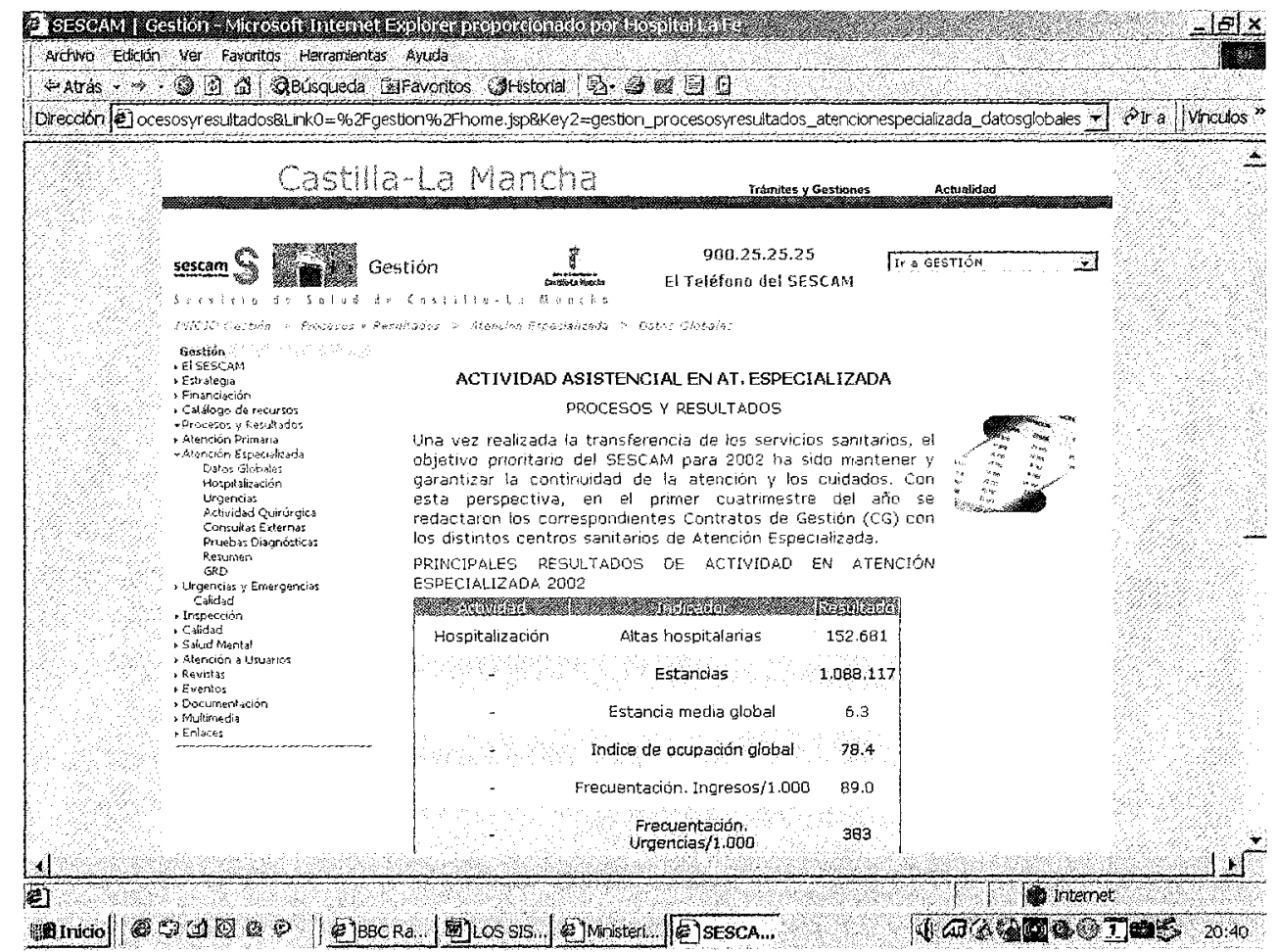

Fig. 5

En estos tres casos se recogen datos disponibles en la página web referentes a la actividad de un servicio regional, de un centro hospitalario y de un área de atención primaria de tres CA diferentes. Con ser un buen ejemplo de indicadores en tres niveles diferentes de la estructura asistencial, si siguiéramos adentrándonos en la información podríamos comprobar que para cada nivel de estructura (servicio de salud, centro hospitalario y atención primaria) la composición de los indicadores no 


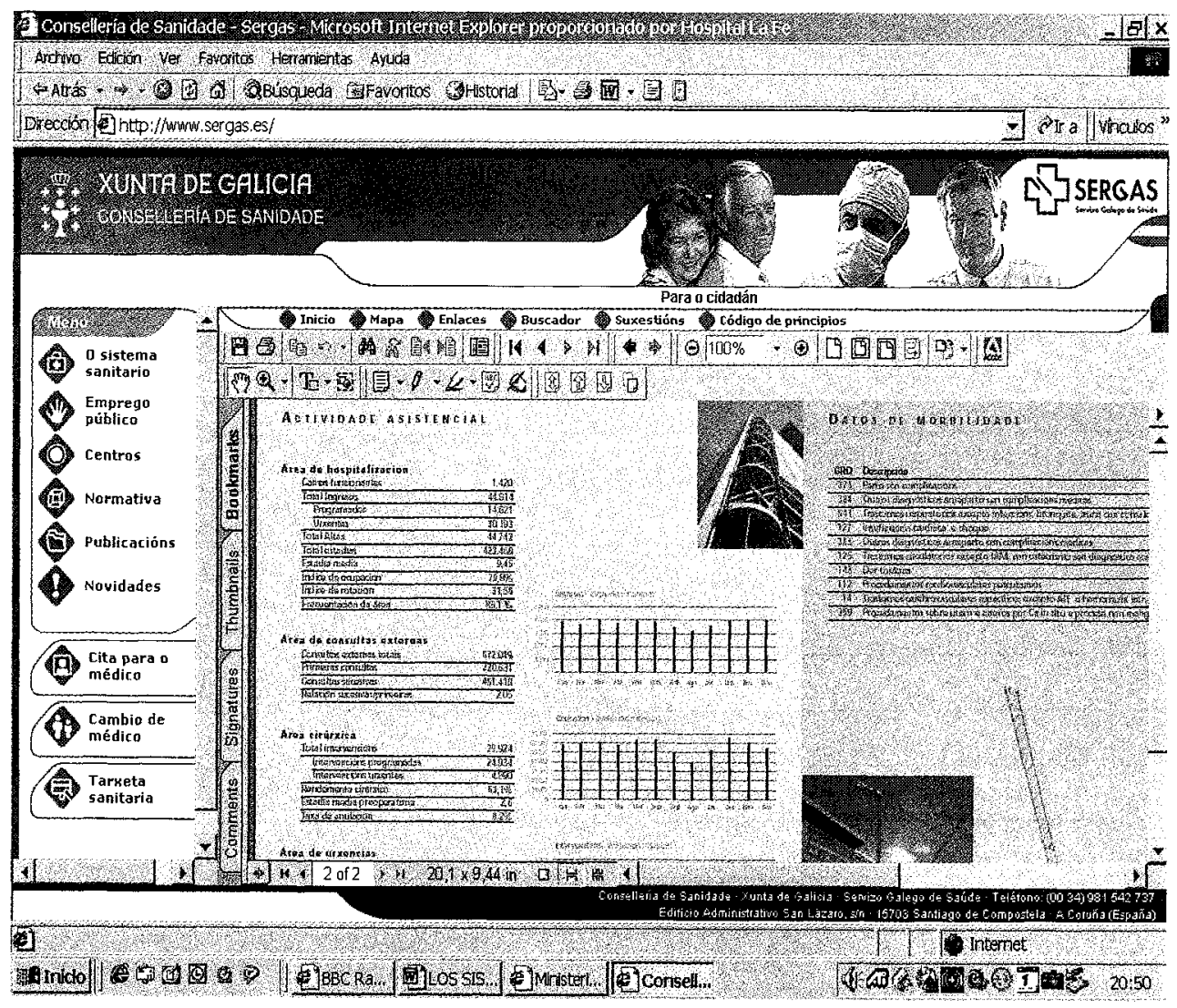

Fig. 6

coincide con lo que, de nuevo, se pierde un aspecto fundamental de la mejora: poder comparar los resultados de cada estructura de una CA con la de otra.

Si volvemos a la website del MSC y accedemos a la página "Los servicios de Autonómicos de Salud» y entramos en la de cualquier CA, en lugar de encontrar indicadores de actividad o resultado o recursos, homogéneos para todas y cada una de las CA y de forma agregada para todo el SNS, que sería lo esperado y deseable, encontramos solamente un link a la website de cada CA que contiene, como hemos comentado anteriormente, información en su mayoría no comparable.

Esto difiere de lo que ocurre en otros SNS en Europa. Si accedemos a la website del National Health Service británico (NHS) podemos ver que para cualquier indicador obtienen datos agregados de todo el SNS 


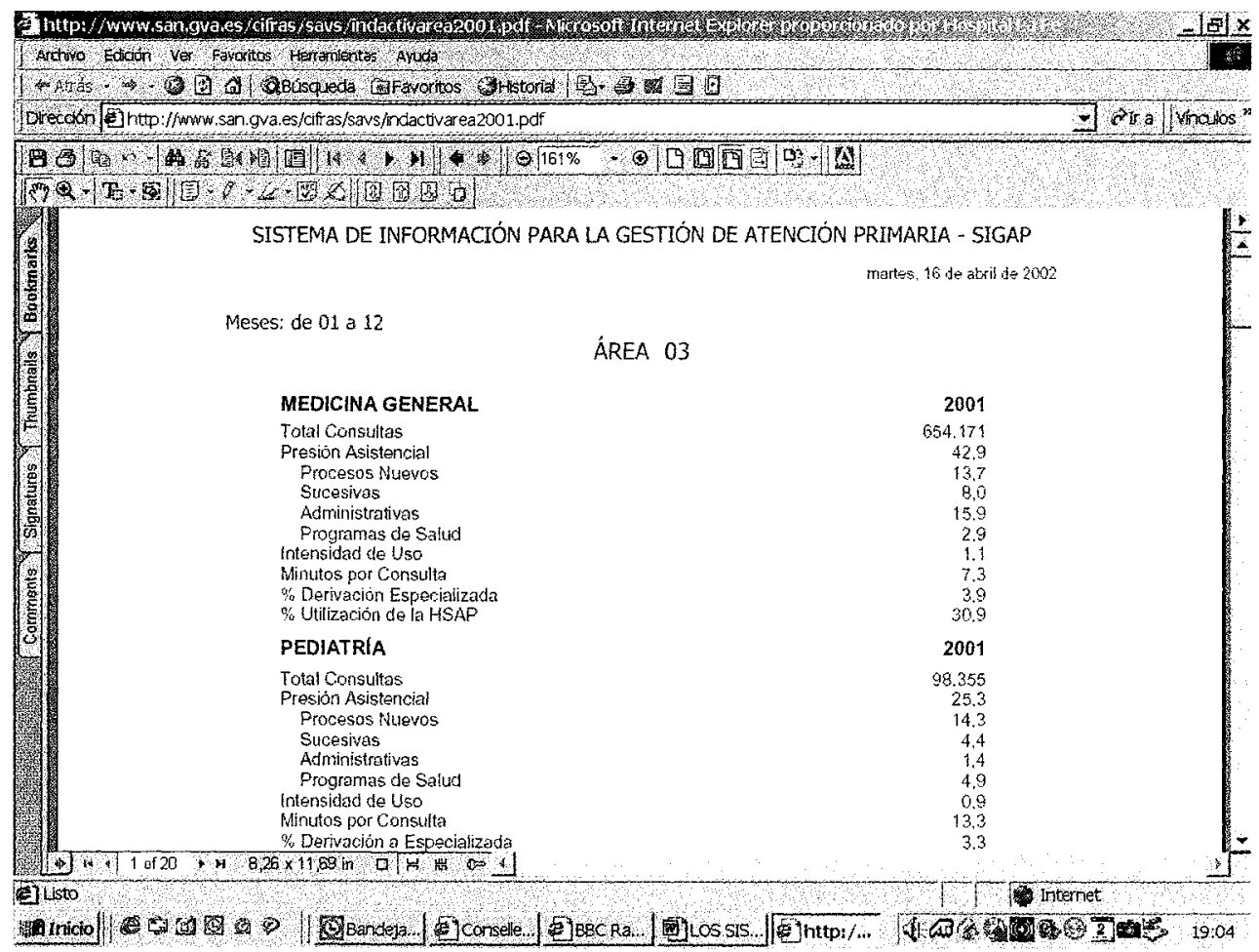

Fig. 7

e incluso, adentrándonos en él, información para cada centro hospitalario.

En la figura 8 podemos observar la demora que existe, por tramos de espera, en primeras visitas hospitalarias para el conjunto de Inglaterra y para cada uno de los hospitales del sistema. Si entramos en cualquiera de ellos (Fig. 9) observamos la espera para cada una de las especialidades de ese centro. En un ejemplo tan paradigmático como las listas de espera vemos como en el caso del NHS se puede obtener una información agregada y desagregada con un amplio nivel de detalle, lo que significa manejar los indicadores de una forma homogénea para todo el SNS, respondiendo, sin duda, a un planteamiento estratégico del cuadro de mandos. Si lo miramos desde la perspectiva del ciudadano la mejora en la información, comparándola con nuestro sistema, es más que evidente. Ejemplos de esta índole marcan el camino a seguir en la mejora de los SIS. 


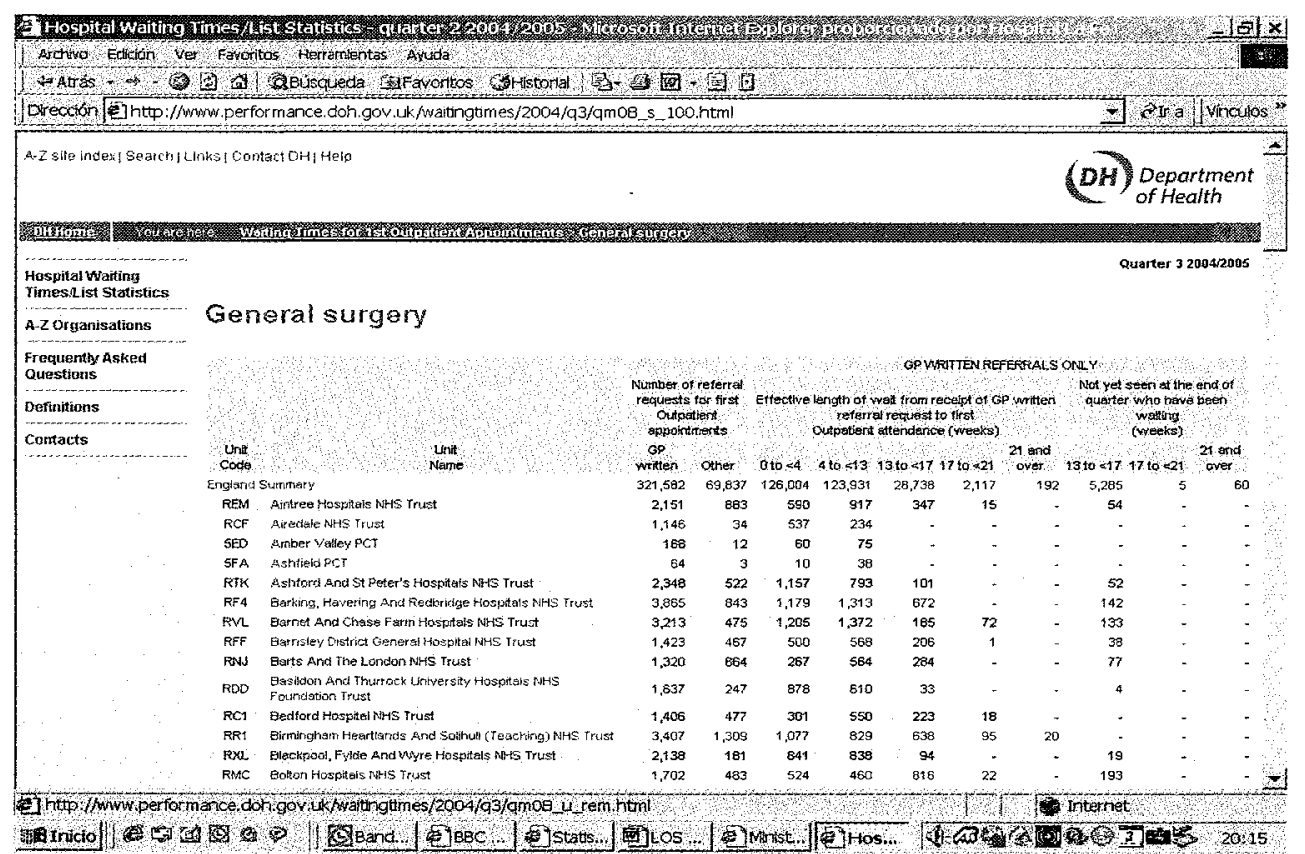

Fig. 8

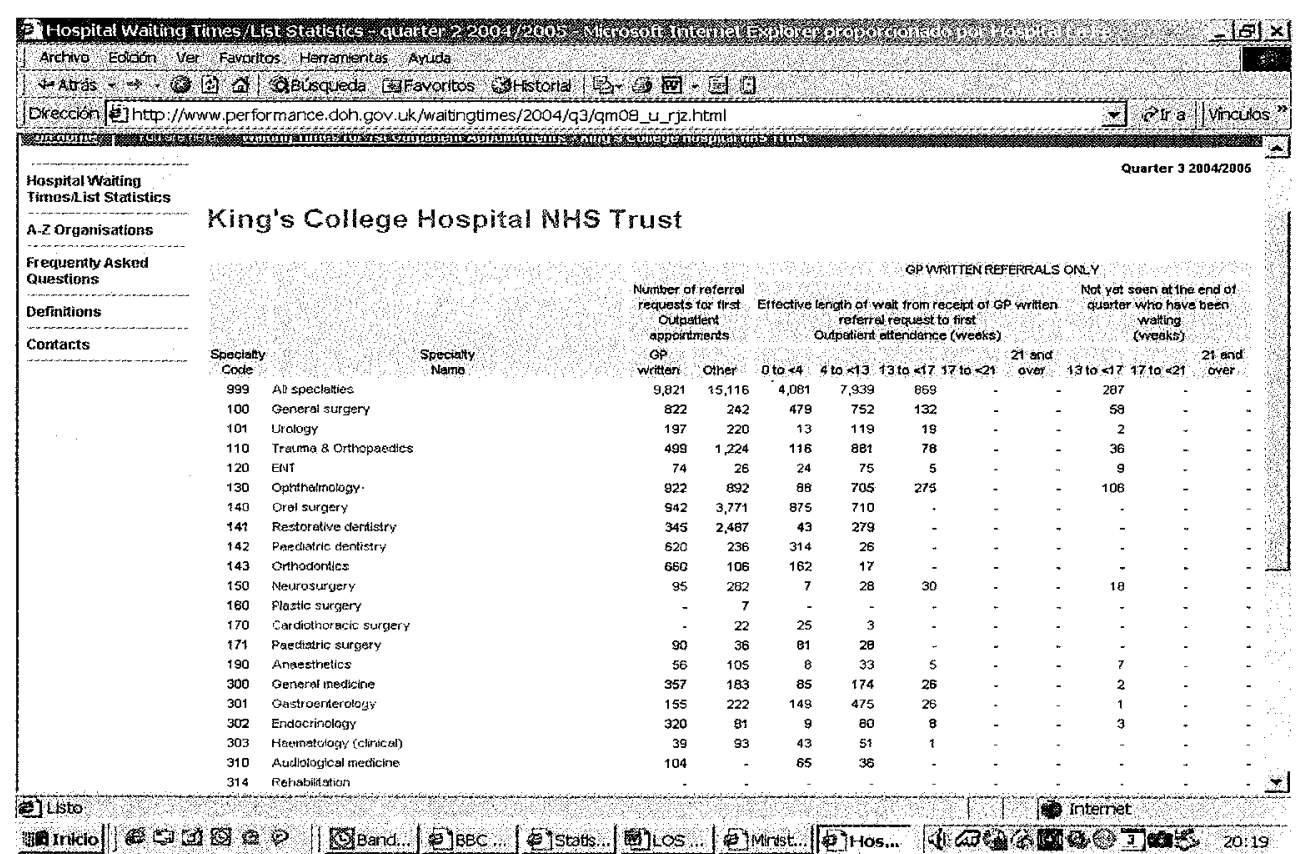

Fig. 9 
Los sistemas de información sanitaria...

\section{Mirando hacia el futuro}

Como hemos podido ver hasta ahora nuestro SIS se caracteriza por manejar una enorme cantidad de datos sin que éstos sean homogéneos, la información ofrecida no es contrastable y los flujos de datos son muy complejos. Por otra parte, el SIS se ha desarrollado desde la óptica de resolver necesidades de información concretas a una determinada entidad, ya sea el MSC o las CA, no valorando las múltiples interrelaciones que existen entre todas ellas.

Si miramos hacia el futuro, el SNS debería incorporar la metodología que están utilizando todas las organizaciones que caminan hacia la excelencia y que no es otra que la utilización de una estrategia común a todo el sistema. Esto permitiría utilizar herramientas potentes para el despliegue de dicha estrategia como el CMI o BSC. El CMI permite orientar toda la organización, en este caso nuestro SNS, hacia la consecución de su visión. Los objetivos estratégicos de la organización recogen, para cada una de las cuatro perspectivas del CMI: Aprendizaje y crecimiento, Procesos Internos, Clientes y Financiera, las iniciativas y acciones para conseguirlos. Para monitorizar el grado de cumplimiento de los objetivos se establece un cuadro de indicadores que son los que transmiten el grado de «salud» del sistema. Los indicadores se convierten así en una herramienta al servicio de la estrategia y responden a las necesidades de los accionistas, en nuestro caso los ciudadanos y las autoridades sanitarias; de los clientes, en nuestro caso los pacientes y de las personas de la organización, en nuestro caso de los profesionales del sistema.

La empresa no es imposible y existen antecedentes de ello en otras partes del mundo. Basta con echar un vistazo al SIS del Gobierno de Ontario (Canadá), elaborado por el Canadian Institute for Health. El contenido del SIS está adaptado a un CMI pues responde a la planificación y despliegue de la estrategia de dicho gobierno. Sus indicadores están distribuidos entre las cuatro perspectivas de su CMI: Integración de sistemas y cambio (se corresponde con la perspectiva de aprendizaje y crecimiento), utilización clínica y resultados (perspectiva de procesos internos), satisfacción de los pacientes (perspectiva de clientes) y resultados financieros (perspectiva financiera). Merece la pena consultar la website: www.hospitalreport.ca/pdf/2003/2003AcFullReportEng.pdf.

La ventaja de utilizar el CMI como sistema de información es que además de informar de la estrategia de una organización lo hace de los resultados que obtiene, tanto de la organización en su conjunto como de las diferentes partes que la componen, resultando aplicable a nuestro SNS de estructura descentralizada. Así, el CMI de todo el SNS y su con- 
junto de indicadores sería el resultado de la agregación de los diferentes CMI de las CA, dándole consistencia, coherencia, homogeneidad y, en de finitiva, calidad a la información.

Para llegar a este grado de madurez del SIS será necesario abordar una política diferente en el seno de las instituciones sanitarias de país, el MSC y las diferentes CA. Esta política deberá gestarse en el Consejo Interterritorial dando cumplimiento a lo recogido en la Ley de Cohesión y Calidad del SNS en su artículo 71 sobre funciones del CI. Lástima que las funciones recogidas en dicho artículo figuren en forma de emitir recomendaciones cuando lo necesario sería obtener compromisos conjuntos de todos los entes que lo componen. De esta forma sería más fácil mantener la hasta ahora buena «salud» de nuestro SNS.

\section{Bibliografía utilizada}

J. Rey del Castillo. Análisis del origen, situación y perspectivas de futuro del proceso de descentralización sanitario español. Javier Rey del Castillo. Rev. Esp. Salud Pública, 1998;72:13-24.

F. Babín Vich. El centro nacional de Información Sanitario como herramienta de apoyo y servicio al Sistema Nacional de Salud. Ponencia realizada en el congreso Inforsalud 1997.

J. L. Monteagudo Peña. La normalización en el sector sanitario. Ponencia realizada en el congreso Inforsalud 1997.

Beatriz González López-Valcárcel et al. Prioridades en la organización de la atención a la salud en España. Informe SESPAS 2002.

Ley $16 / 2003$, de 28 de mayo, de cohesión y calidad del Sistema Nacional de Salud. B.O.E $\mathrm{n}^{0}$ 128:20567-20588 de 29 de mayo de 2003.

L. Bohigas Santasusagna. La calidad, un objeto cohesionador del Sistema Nacional de Salud. El Médico, Anuario 2004, Pág. 22-24.

J. Carnicero et al. Conclusiones. Informe SEIS. De la historia clínica a la historia de salud electrónica. Editado por SEIS. Madrid 2003.

M. Humber. Nacional programme for information technology. BMJ 2004;328:1145-6.

N. M. Lorenci. Beyond the gadgets. BMJ 2004;328:1146-7.

www.msc.es

www.sescam.jcem.es

www.san.gva.es

www.sergas.es

www.performance.doh.gov.uk

www.hospitalreport.ca

R. Kaplan, D. Norton. Cuadro de Mando Integral. $2^{a}$ edición. Editorial Gestión 2000. Barcelona 2000. 